\title{
Local approximation to the critical parameters of quantum wells
}

\author{
Francisco M Fernández ${ }^{1}$ and Javier Garcia \\ INIFTA (UNLP, CCT La Plata-CONICET), División Química Teórica, Blvd. 113 \\ S/N, Sucursal 4, Casilla de Correo 16, 1900 La Plata, Argentina
}

\begin{abstract}
We calculate the critical parameters for some simple quantum wells by means of the Riccati-Padé method. The original approach converges reasonably well for nonzero angular-momentum quantum number $l$ but rather too slowly for the s states. We therefore propose a simple modification that yields remarkably accurate results for the latter case. The rate of convergence of both methods increases with $l$ and decreases with the radial quantum number $n$. We compare RPM results with WKB ones for sufficiently large values of $l$. As illustrative examples we choose the onedimensional and central-field Gaussian wells as well as the Yukawa potential. The application of perturbation theory by means of the RPM to a class of rational potentials yields interesting and baffling unphysical results.
\end{abstract}

\section{Introduction}

The accurate calculation of the number of bound states supported by a finite quantum-mechanical potential well is of great physical and mathematical importance and for this reason there has been considerable interest in

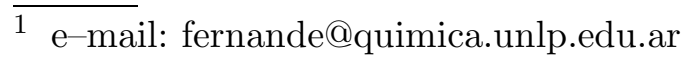


the derivation of upper and lower bounds [1-13]. Most of those bounds are given in terms of the potential-energy function. In a recent paper Liverts and Barnea [14] proceeded in a different way and proposed the calculation of the critical parameters for negative central-field quantum wells. To this end they applied two exact methods and the WKB approach, the latter for the estimation of the large-quantum number behaviour of the critical parameters. In this context a critical parameter is the value of a potential parameter for which an energy eigenvalue is exactly zero (what the authors call a transition state). As they pointed out, one can obtain the exact number of bound states from the tables of critical parameters, as well as other relevant information about the eigenvalue equation [14].

There are local and global methods for the calculation of eigenvalues and eigenfunctions. The former are based on the behaviour of the solution at a properly chosen coordinate point; for example, a power-series expansion. On the other hand, global approaches like the variational method take into account the whole coordinate interval (through expectation values of the associated linear operators, etc.). In principle, local methods are expected to be unsuitable for the calculation of critical parameters. Even the Riccati-Padé method (RPM) [15, 16], based on Padé approximants, was shown to be impractical for the calculation of the eigenvalues of the Yukawa potential close to the zero-energy threshold (transition state) [15]. The purpose of this paper is to investigate in more detail whether those earlier results already prove that the RPM is actually useless for the calculation of critical parameters.

In section 2 we outline the main ideas of the RPM. In section 3 we briefly discuss the solutions of the Schrödinger equation with even-parity potential wells. In section 4 we apply the approach to some simple one-dimensional models: the Pöschl-Teller potential, the Gaussian well and a rational potential. We calculate some critical parameters and the corresponding eigenfunctions for the first and third cases. In section 5 we propose a modified version of 
the RPM that is more suitable for the calculation of critical parameters and apply it to the Gaussian well. Some of those results also apply to s-states of the analogous central-field model. In section 6 we apply the modified RPM to the s-states of central-field models and choose the Yukawa potential as a suitable illustrative example. We also show that the original RPM is suitable for the calculation of critical parameters of states with $l>0$. In section 7 we discuss the application of perturbation theory to a model with a rational potential that is exactly solvable at threshold. In this case we discuss the appearance of spurious RPM eigenvalues. Finally, in section 8 we summarize the main results and draw conclusions.

\section{The Riccati-Padé method}

We consider the eigenvalue equation

$$
\psi^{\prime \prime}(x)+Q(E, x) \psi(x)=0,-\infty<x<\infty
$$

were $E$ is the eigenvalue. We assume that $\psi(x)$ can be expanded about the origin as

$$
\psi(x)=x^{s} \sum_{j=0}^{\infty} c_{j} x^{\beta j}, \alpha, \beta>0 .
$$

It is clear that

$$
f(x)=\frac{s}{x}-\frac{\psi^{\prime}(x)}{\psi(x)},
$$

can be expanded about the origin as

$$
f(x)=x^{\beta-1} \sum_{j=0}^{\infty} f_{j} z^{j}, z=x^{\beta} .
$$


We approximate $f(x)$ by means of a rational function of the form $x^{\beta-1}[M / N](z)$ where

$$
[M / N](z)=\frac{\sum_{j=0}^{M} a_{j} z^{j}}{\sum_{j=0}^{N} b_{j} z^{j}}=T(M+N+1, z)+O\left(z^{M+N+2}\right)
$$

and

$$
T(n, z)=\sum_{j=0}^{n} f_{j} z^{j}
$$

We choose $M \geq N$ and define $d=M-N$. It is not possible to satisfy the condition (5) unless $H_{D}^{d}=\left|f_{i+j+d-1}\right|_{i, j=1}^{D}=0, D=N+1$. The coefficients $f_{j}$, and thereby the Hankel determinant $H_{D}^{d}$, depend on the eigenvalue $E$. Some of the roots $E^{[D, d]}$ of $H_{D}^{d}(E)=0$ converge toward the eigenvalues of Eq. (1) as $D$ increases [15, 16].

The ordinary Padé approximation to $f(x)$ is

$$
[M / N](z)=\frac{\sum_{j=0}^{M} a_{j} z^{j}}{\sum_{j=0}^{N} b_{j} z^{j}}=T(M+N, z)+O\left(z^{M+N+1}\right) .
$$

If $z_{0}$ is a zero of the denominator then

$$
z_{0}=-\frac{b_{N-1}}{b_{N}}-\frac{b_{N-2}}{b_{N} z_{0}}-\ldots-\frac{b_{0}}{b_{N} z_{0}^{N-1}}
$$

Suppose that $E^{*}$ is a root of $b_{N}(E)=0$ and that $b_{N-1}(E)$ does not vanish in the interval $\left(E^{*}-\epsilon, E^{*}+\epsilon\right)$ for a sufficiently small positive real number $\epsilon$. Therefore $\left|z_{0}\right| \rightarrow \infty$ as $E \rightarrow E^{*}$. The coefficient $b_{N}$ is proportional to the Hankel determinant $H_{N-1}^{d+1}(E)$ so that the Hankel condition $H_{D}^{d}(E)=0$ is equivalent to moving a singularity of a rational approximation towards infinity [17]. It is also equivalent to moving a zero of the approximate $\psi(x)$ towards infinity. Consequently, it appears to be reasonable to assume that the Hankel condition is equivalent to selecting bound states that vanish at infinity. 
The strategy just outlined applies to other nonlinear equations and for this reason Amore and Fernández [18] chose the more general name Padé-Hankel method which was later discussed by Abbasbandy and Bervillier [17]. However, for historical reasons we prefer to keep the original name RPM when the problem is a Riccati equation derived from the Schrödinger one [15, 16].

\section{Parity-invariant finite wells}

The Riccati-Padé method is known to produce accurate eigenvalues for infinite wells or sufficiently deep finite ones [15, 16]. The purpose of this section is to investigate to which extent it is possible to apply the RPM to shallow wells. To this end we consider the eigenvalue equation (1) with

$$
Q(E, x)=2[E-V(x)]
$$

where the potential-energy function $V(x)$ exhibits a minimum at $V(0)<0$ and $V(x \rightarrow \pm \infty)=0$. In order to simplify the discussion we assume that $V(-x)=V(x)$ so that

$$
Q(E, x)=\sum_{j=0}^{\infty} Q_{j} x^{2 j} .
$$

The shape of a parity-invariant potential is commonly determined by a smaller

number of parameters. In addition to it, the results for the odd states also apply to the solutions of the Schrödinger equation with a central-field potential having zero angular momentum quantum number $l$ (s states).

The eigenfunctions of the Hamiltonian operator with a parity-invariant potential are even or odd; therefore, $s=0$ for the former, $s=1$ for the latter and $\beta=2$ in both cases. We thus have

$$
f(x)=x \sum_{j=0}^{\infty} f_{j} x^{2 j},
$$


where the first coefficients are

$$
\begin{aligned}
& f_{0}=\frac{Q_{0}}{1+2 s}, \\
& f_{1}=\frac{Q_{0}^{2}}{(3+2 s)(1+2 s)^{2}}+\frac{Q_{1}}{3+2 s}, \\
& f_{2}=\frac{2 Q_{0}^{3}}{(5+2 s)(3+2 s)(1+2 s)^{3}}+\frac{2 Q_{0} Q_{1}}{(5+2 s)(1+2 s)(3+2 s)}+\frac{Q_{2}}{(5+2 s)} .
\end{aligned}
$$

Besides, the function $f(x)$ is a solution to the Riccati equation

$$
f^{\prime}(x)+\frac{2 s}{x} f(x)-f(x)^{2}-Q(E, x)=0 .
$$

For convenience we define $v_{0}=-V(0)>0$ and $v(x)=-V(x) / v_{0}$. For all values of the well depth $v_{0}>0$ there is always a bound ground state with energy $E_{0}$. The number of bound states with energies $E_{0}<E_{1}<\ldots<$ $E_{n}<0$ depends on $v_{0}$. As $v_{0}$ decreases the highest bound-state energy, say $E_{n}$, approaches the threshold $E=0$ from below and we have a critical well parameter $v_{0, n}$ when $E_{n}=0$. Consequently, there are $n+1$ bound states when $v_{0, n}<v_{0}<v_{0, n+1}$.

We assume that $V(x)$ vanishes faster than $x^{-2}$ as $|x| \rightarrow \infty$; that is to say

$$
\lim _{|x| \rightarrow \infty} x^{2} V(x)=0
$$

Therefore, an eigenfunction for the arbitrary energy $E<0$ behaves asymptotically as

$$
\psi(x) \sim A\left(E, v_{0}\right) e^{-\alpha x}+B\left(E, v_{0}\right) e^{\alpha x},|x| \rightarrow \infty
$$

where $\alpha=\sqrt{-2 E}$. The bound states are given by the condition $B\left(E_{j}, v_{0}\right)=0$ that leads to square-integrable eigenfunctions. 
When $E=0$ the two asymptotic solutions in Eq. (15) are linearly dependent.

In this case the general solution to $\psi^{\prime \prime}(x)=0$ behaves as

$$
\psi(x) \sim A\left(v_{0}\right)+B\left(v_{0}\right) x, E=0,|x| \rightarrow \infty .
$$

The solution at threshold is not square integrable but we can think of it as

the limit of a square integrable one $\lim _{E \rightarrow 0} A\left(E, v_{0}\right) e^{-\alpha x}=A\left(v_{0}\right)(|x| \rightarrow \infty)$. Therefore, the critical parameters are roots of $B\left(v_{0, n}\right)=0$ and the boundary condition at threshold is

$$
\lim _{|x| \rightarrow \infty} \psi^{\prime}(x)=0, E=0
$$

\section{Examples}

In what follows we discuss some simple model potentials to illustrate the application of the RPM.

\subsection{Modified Pöschl-Teller potential}

As a first example we consider the modified Pöschl-Teller potential

$$
V(x)=-\frac{v_{0}}{\cosh ^{2}(x)}, v_{0}>0
$$

There are two reasons for this choice: first, we can solve the Schrödinger equation and obtain a simple expression for the eigenvalues: [19]

$$
\begin{aligned}
E_{n} & =-\frac{(\lambda-n-1)^{2}}{2}, n=0,1, \ldots \\
\lambda & =\frac{1+\sqrt{1+8 v_{0}}}{2}
\end{aligned}
$$


Second, the exact bound-state solutions are hypergeometric functions of $y=$ $\cosh ^{2} x$ so that the RPM based on an $x$-power series can only yield approximate results. Therefore, this model is a suitable benchmark for testing the performance of the approach.

As discussed above the critical values of the potential parameter $v_{0}$ are determined by the condition $E_{n}\left(v_{0, n}\right)=0$. It follows from equation (19) that $v_{0, n}=n(n+1) / 2, n=0,1, \ldots$ We first investigate if there are converging sequences of roots $E^{[D, d]}$ of the Hankel determinant $H_{D}^{d}$ as $v_{0}$ approaches $v_{0,0}=0$ and $v_{0,2}=3$. We calculated $E^{[D, 0]}, D=2,3, \ldots$ for $v_{0}=v_{0, n}+10^{-k}$, for $k=1,2, \ldots$. The results show that there are convergent sequences of roots for $D=D_{k}, D_{k}+1, \ldots$ and that the starting point of each sequence $D_{k}$ increases as $k$ increases. There seems to be convergent sequences no matter how large the value of $k$. In other words, the RPM appears to be successful no matter how close $v_{0}$ is to the critical value $v_{0, n}$.

Since the roots of the Hankel determinants $H_{D}^{d}\left(E, v_{0}\right)=0$ give rise to sequences that clearly converge towards the eigenvalues $E_{n}\left(v_{0}\right)$ for $v_{0}$ quite close to $v_{0, n}$, then one would expect to find sequences of roots of $H_{D}^{d}\left(E=0, v_{0}\right)=0$ that converge towards the critical parameters $v_{0, n}$. This is exactly the case for this model and one obtains the critical parameters with any desired degree of accuracy with Hankel determinants of relatively small dimension. There are, however, two surprising facts. The first one is that the RPM yields all the critical parameters $v_{0, n}, n=1,2, \ldots$ when choosing either the even $(s=0)$ or odd $(s=1)$ functions. One would expect the even or odd values of $n$ to appear separately with even or odd functions, respectively. The second surprising fact is that the RPM with $s=0$ yields the critical parameters with odd $n$ more accurately than those with even $n$. The opposite situation takes place when choosing $s=1$. 
We can understand the occurrence of twice as much critical parameters as expected by obtaining the corresponding wave functions in the usual way [19]. If $\psi_{n, s}(x)$ denotes the solution of parity $s$ for $v_{0}=v_{0, n}$ and $E=0$, then the first of them are given by

$$
\begin{aligned}
& \psi_{1,0}(x)=\frac{2 x}{e^{2 x}+1}-x+1, \\
& \psi_{1,1}(x)=1-\frac{2}{e^{2 x}+1}, \\
& \psi_{2,0}(x)=\frac{2\left(4 e^{2 x}-e^{4 x}-1\right)}{e^{4 x}+2 e^{2 x}+1}, \\
& \psi_{2,1}(x)=-\frac{\left[e^{4 x}(2 x-3)-8 x e^{2 x}+2 x+3\right]}{4\left(e^{4 x}+2 e^{2 x}+1\right)} .
\end{aligned}
$$

We appreciate that $\psi_{1,1}(x)$ and $\psi_{2,0}(x)$ are convergent while $\psi_{1,0}(x)$ and $\psi_{2,1}(x)$ are divergent. In general, $\psi_{n, s}$ is convergent or divergent provided that $n+s$ is even or odd, respectively:

$$
\begin{aligned}
\lim _{|x| \rightarrow \infty} \psi_{n, s,}(x) & =A, n+s=2 k, \\
\lim _{|x| \rightarrow \infty} x^{-1} \psi_{n, s}(x) & =B, n+s=2 k-1, \\
k & =1,2, \ldots
\end{aligned}
$$

We conclude that the RPM approaches both the convergent and divergent solutions for this problem when $E=0$. This is the reason why the whole set of critical parameters $v_{0, n}$ appears for both the even and odd solutions: half of them are convergent and the other half divergent. It is clear, as already argued above, that the RPM does not yield the exact result because the exact $f(x)$ is not a rational function of $x$ for any of the functions (20).

It is not clear to us why the RPM with $s=0(s=1)$ yields the critical parameters with odd (even) $n$ more accurately. We will discuss this point with somewhat more detail below by means of a solvable model with a rational potential. 


\subsection{Gaussian well}

The Gaussian well

$$
V(x)=-v_{0} e^{-x^{2}}
$$

is another suitable choice because the potential is extremely simple but the Schrödinger equation is not exactly solvable. In this case the behaviour of the sequences of roots of $H_{D}^{d}\left(E, v_{0}\right)=0$ appears to be similar except that the starting point $D_{k}$ of a given sequence increases more pronouncedly as $v_{0}$ approaches $v_{0, n}$ and we could not find converging sequences of roots of $H_{D}^{d}\left(E=0, v_{0}\right)=0$. It is not clear to us which is the feature of this well that makes such a difference. Since the present form of the RPM appears to be unsuitable for obtaining the critical parameters for this problem in section 5 we will discuss an improved version of the approach.

\subsection{Rational potential}

The third example in this section is the potential well

$$
V(x)=-\frac{v_{0}}{\left(1+x^{2}\right)^{2}}
$$

that satisfies the condition (14). Joseph [20] studied the family of central-field potentials $V(r)=-\lambda r^{\alpha-2}\left(r_{0}^{2}+r^{2}\right)^{-\alpha}$ in his discussion of local degeneracy. Clearly, the potential (23) is the one-dimensional version of the case $\alpha=2$. Besides, present results for the odd states should agree with those obtained by Joseph for $\alpha=2$ and $l=0$.

The roots of the Hankel determinants $H_{D}^{0}\left(E=0, v_{0}\right)$ yield exact critical parameters $v_{0, n}=n(n+2) / 2, n=1,2, \ldots$. These results correspond to exact 
rational solutions to the Riccati equation (13). In order to understand their meaning we construct the corresponding wavefunctions as

$$
\psi_{n, s}=x^{s} \exp \left[-\int f(x) d x\right]
$$

The first even and odd ones are

$$
\begin{aligned}
& \psi_{1,0}(x)=\frac{1-x^{2}}{\sqrt{x^{2}+1}}, \\
& \psi_{2,0}(x)=\frac{1-3 x^{2}}{x^{2}+1}, \\
& \psi_{3,0}(x)=\frac{\left(x^{2}+2 x-1\right)\left(x^{2}-2 x-1\right)}{\left(1+x^{2}\right)^{3 / 2}}, \\
& \psi_{4,0}(x)=\frac{5 x^{4}-10 x^{2}+1}{\left(1+x^{2}\right)^{2}},
\end{aligned}
$$

and

$$
\begin{aligned}
& \psi_{1,1}(x)=\frac{x}{\sqrt{1+x^{2}}}, \\
& \psi_{2,1}(x)=\frac{x\left(x^{2}-3\right)}{1+x^{2}}, \\
& \psi_{3,1}(x)=\frac{x\left(x^{2}-1\right)}{\left(1+x^{2}\right)^{3 / 2}}, \\
& \psi_{4,1}(x)=\frac{x\left(x^{4}-10 x^{2}+5\right)}{\left(1+x^{2}\right)^{2}},
\end{aligned}
$$

respectively. As in the case of the modified Pöschl-Teller potential the solutions $\psi_{n, s}$ are convergent or divergent provided that $n+s$ is even or odd, respectively; more precisely, they satisfy equations (21). According to the discussion of section $3 \psi_{2 k, 0}(x)$ are the even solutions to the Schrödinger equation for $E=0$ and $v_{0}=2 k(k+1)$ (satisfy the condition $\left.B\left(v_{0}\right)=0\right)$. On the other hand, $\psi_{2 k-1,1}(x)$ are the odd solutions for $E=0$ and $v_{0}=\left(4 k^{2}-1\right) / 2, k=$ $1,2, \ldots$ The latter agree with Joseph's ones when $\lambda=2 v_{0}[20]$. The remaining solutions $\psi_{2 k, 1}(x)$ and $\psi_{2 k-1,0}(x)$ are the unphysical divergent solutions to the Schrödinger equation. We see that the RPM yields the exact convergent and divergent solutions to the Schrödinger equation with the potential (23) when 
$E=0$. It is worth noting that the RPM does not distinguish between physical an unphysical results unless one manages to obtain the wavefunction from its logarithmic derivative as we did it in this example. In other cases, like the potential (18), it may be easier to resort to another approach to obtain the wavefunction from the parameters given by the RPM.

In the appendix we solve the Schrödinger equation for this potential and derive the exact convergent and divergent solutions for $E=0$.

Although the RPM yields the exact critical parameters it is not suitable for the calculation of the energies close to threshold. The sequence of roots of $H_{D}^{d}\left(E, v_{0}\right)=0$ converge rather too slowly when $v_{0}$ is close (slightly greater than) a critical parameter. When $E<0$ the function $f(x)$ is not an exact rational function and the RPM yields approximately those eigenvalues that are not too close to threshold. We calculated the ground state for $v_{0}=3 / 2$ $\left(E_{1}=0\right)$ and the first two bound states for $v_{0}=4\left(E_{2}=0\right)$. In the three cases we found that the sequences $E^{[D, 0]}$ converge from above and the sequences $E^{[D, 1]}$ from below. We assume that the former provides upper bounds and the latter lower ones as in an earlier treatment of the quartic anharmonic oscillator [15, 16]. Thus, from sequences of roots with $D \leq 80$ we conjecture that

$$
\begin{aligned}
& -0.6985262171667534202327>E_{0}>-0.6985262171667534202332, v_{0}=\frac{3}{2} \text {, } \\
& -2.4713450252412636948012742>E_{0}>-2.4713450252412636948012743, v_{0}=4 \text {, } \\
& -0.42640598980647065078>E_{1}>-0.42640598980647065113, v_{0}=4 \text {. }
\end{aligned}
$$

We are not aware of any calculation of the eigenvalues and eigenfunctions for this rational potential. 


\section{$5 \quad$ Modified RPM for critical parameters}

According to the results of section 3 the appropriate boundary condition at threshold is given by equation (17). Therefore, it seems reasonable to look for an ansatz with poles at the zeros of $\psi^{\prime}(x)$. One suitable choice is the function

$$
g(x)=\frac{1-s}{x}-\frac{\psi^{\prime \prime}(x)}{\psi^{\prime}(x)}
$$

We thus have

$$
\frac{1-s}{x} f(x)-f(x) g(x)+\frac{s}{x} g(x)=Q(E, x)
$$

and

$$
g(x)=x \sum_{j=0}^{\infty} g_{j} x^{2 j}
$$

The first coefficients are

$$
\begin{aligned}
& g_{0}=\frac{Q_{0}}{3}-\frac{2 Q_{1}}{3 Q_{0}} \\
& g_{1}=\frac{Q_{0}^{2}}{45}-\frac{4 Q_{2}}{5 Q_{0}}+\frac{2 Q_{1}^{2}}{9 Q_{0}^{2}}+\frac{11 Q_{1}}{45}
\end{aligned}
$$

for $s=0$ and

$$
\begin{aligned}
g_{0} & =Q_{0}, \\
g_{1} & =Q_{1}+\frac{1}{3} Q_{0}^{2}, \\
g 2 & =Q_{2}+\frac{8}{15} Q_{0} Q_{1}+\frac{2}{15} Q_{0}^{3},
\end{aligned}
$$

for $s=1$. We apply the RPM exactly in the same way and construct the Han-

kel determinants with the coefficients $g_{j}: H_{D}^{d}\left(E, v_{0}\right)=\left|g_{i+j+d-1}\left(E, v_{0}\right)\right|_{i, j=1}^{D}=$ 0. 
We obtain convergent sequences of roots of $H_{D}^{d}\left(E=0, v_{0}\right)=0$ for all the models discussed above. In particular, Table 1 shows the first critical parameters for the Gaussian well estimated from the roots of the Hankel determinants with $D \leq 80, d=0$ and $d=1$. For comparison we add the results of Liverts and Barnea [14] for the s-states of the central-field model. The critical parameters for the central-field model with angular momentum quantum number $l=0$ are exactly those for the odd states of the one-dimensional case.

In closing this section we mention that we also tried the alternative ansatz $\psi(x) / \psi^{\prime}(x)$ for odd eigenfunctions but in this case the rate of convergence proved to be considerably lower.

\section{Central-field models}

The results of section 5 suggest that the present approach may also be suitable for the s-states of other central-field models. Although the present paper is focused on one-dimensional parity-invariant models we can outline a strategy for the treatment of central-field models. We write the radial part of the dimensionless Schrödinger equation as

$$
\begin{aligned}
& \psi^{\prime \prime}(r)+\left[Q(r)-\frac{l(l+1)}{r^{2}}\right] \psi(r)=0, \\
& Q(r)=2[E-V(r)], \psi(0)=0,
\end{aligned}
$$

and assume that

$$
Q(r)=\sum_{j=-1}^{\infty} Q_{j} r^{j}
$$

As in earlier papers we define [15]

$$
f(r)=\frac{l+1}{r}-\frac{\psi^{\prime}(r)}{\psi(r)},
$$


and in order to apply the modified RPM to the calculation of critical parameters we resort to the auxiliary function

$$
g(r)=\frac{l}{r}-\frac{\psi^{\prime \prime}(r)}{\psi^{\prime}(r)}
$$

They are related by

$$
\frac{l+1}{r} g(r)-f(r) g(r)+\frac{l}{r} f(r)-Q(r)=0
$$

and can be expanded in a Taylor series about the origin as

$$
\begin{aligned}
& f(r)=\sum_{j=0}^{\infty} f_{j} r^{j}, \\
& g(r)=\sum_{j=0}^{\infty} g_{j} r^{j} .
\end{aligned}
$$

As an illustrative example we choose the Yukawa potential

$$
V(r)=-\frac{v_{0} e^{-r}}{r}
$$

and show the results in Table 2 for the first s-states estimated from roots of the $g$-Hankel determinants with $D \leq 80, d=0$ and $d=1$. Present results agree with those of Liverts and Barnea [14] and Singh and Varshni [21] up to the last digit reported by them.

The asymptotic behaviour of the solutions to the central-field models when $E=0$ is given by

$$
\psi(r) \sim A r^{-l}+B r^{l+1}
$$

Therefore, we expect that the original RPM yields reasonable critical parameters for $l>0$. In other words, the roots of the $f$-Hankel determinants are expected to approach the roots of $B\left(v_{0, n, l}\right)=0$ as the determinant dimension 
increases. Tables 3 and 4 clearly show that the rate of convergence of the RPM increases with $l$ and decreases with $n$.

Since the accuracy of the RPM increases with $l$ we can test the WKB large$l$ asymptotics $\beta_{n, l} \sim \operatorname{el}(l+1)$ derived by Liverts and Barnea [14] for both the Yukawa and Gaussian potentials (note that $\beta_{n, l}=2 v_{0, n, l}$ ). We can also compare these results with the variational estimates

$$
v_{0,1, l}^{Y}=\frac{2^{2 l}(l+1)^{2 l+3}}{(2 l+1)^{2 l+1}},
$$

and

$$
v_{0,1, l}^{G}=\frac{(2 l+3)^{\frac{2 l+5}{2}}}{8(2 l+1)^{\frac{2 l+1}{2}}},
$$

derived by means of the trial functions $\varphi(r)=N r^{l+1} e^{-a r}$ and $\varphi(r)=N r^{l+1} e^{-a r^{2}}$ for the Yukawa and Gaussian potentials, respectively [25].

Tables 5 and 6 show the RPM, WKB and variational results, as well as the logarithmic errors of the two latter ones. We appreciate that the variational estimates are somewhat more accurate but the WKB expression shows the striking fact that the large- $l$ asymptotic behaviour for the critical parameters for both potentials is exactly the same. Although the two variational results are different for small and moderate $l$ they agree with the WKB ones for sufficiently large $l$ :

$$
\lim _{l \rightarrow \infty} \frac{v_{0,1, l}^{Y}}{l(l+1)}=\lim _{l \rightarrow \infty} \frac{v_{0,1, l}^{G}}{l(l+1)}=\frac{e}{2} .
$$

The RPM results in tables [5] and [6] are accurate to the last digit and were obtained by means of Hankel determinants of dimension as small as $D=10$.

The rate of convergence of the modified RPM based on $g$-Hankel determinants also increases with $l$ but we do not deem necessary to show those results. 
Although present results are more accurate than those of Liverts and Barnea [14] and Singh and Varshni [21] one should not conclude that the RPM is superior to the approaches developed by those authors. Those other methods are more general because present local approximation is based on the Taylor expansion of the solution about some chosen point which limits the class of potentials that can be treated successfully. However, the RPM is a straightforward simple approach that applies to a wide variety of problems. In many cases it yields quite accurate results and may be suitable for testing other approaches and even for setting benchmark data.

\section{Perturbation theory about the threshold}

We can expand the exact energy (19) for the modified Pöschl-Teller potential in a Taylor series about $v_{0, n}$ and obtain the perturbation series about the threshold

$$
\begin{aligned}
E_{n} & =-\frac{2 \xi^{2}}{(2 n+1)^{2}}+\frac{8 \xi^{3}}{(2 n+1)^{4}}-\frac{40 \xi^{4}}{(2 n+1)^{6}}+\frac{224 \xi^{5}}{(2 n+1)^{8}}+O\left(\xi^{6}\right), \\
\xi & =v_{0}-v_{0, n}
\end{aligned}
$$

that converges for all $|\xi|<(2 n+1)^{2} / 8$. Note that the perturbation correction of first order is zero for all states and that we obtain a negative energy for both $v_{0}>v_{0, n}$ and $v_{0}<v_{0, n}$ if $\xi$ is sufficiently small, in spite of the fact that the $n$-th state moves into the continuum in the latter case. We can carry out a similar calculation for models that are not exactly solvable by means of the RPM. In what follows we illustrate the strategy by means of the apparently most favourable case of the rational potential (23) for which the RPM yields the exact solution at threshold.

The roots of a Hankel determinant $H_{D}^{d}\left(E, v_{0}\right)=0$ give us approximations to 
either $E\left(v_{0}\right)$ or $v_{0}(E)$. If we substitute

$$
E=E^{(1)} \xi+E^{(2)} \xi^{2}+\ldots+E^{(k)} \xi^{k}
$$

and $v_{0}=v_{0, n}+\xi$ into the Hankel determinant, then we can obtain the coefficients $E^{(j)}, j=1,2, \ldots k$ of the perturbation series, the accuracy increasing with $D$. Based on the Hellmann-Feynman theorem [22] (see also [23] for a discussion about degenerate states)

$$
\frac{d E}{d v_{0}}=-\left\langle\frac{1}{\left(1+x^{2}\right)^{2}}\right\rangle,
$$

we expect that

$$
E^{(1)}=\lim _{\xi \rightarrow 0^{+}} \frac{d E}{d \xi} \leq 0,
$$

for a physically acceptable solution. Since the solutions are not square integrable when $\xi=0$ then the expectation value in equation (46) is meaningless at threshold; however the limit (47) may hopefully be finite. In fact, $E^{(1)}=0$ for the Pöschl-Teller potential.

It follows from the discussion in the subsection 4.3 that $E_{1}=0$ when $v_{0}=3 / 2$. However, if we substitute $v_{0}=3 / 2+\xi$ and the series (45) into the Hankel determinants for $s=1$ we obtain the unphysical result

$$
E=\frac{1}{8} \xi-\frac{7}{64} \xi^{2}+\frac{29}{768} \xi^{3}-\frac{1847}{184320} \xi^{4}+\frac{275357}{77414400} \xi^{5}+O\left(\xi^{6}\right) .
$$

According to this expansion the energy increases as $v_{0}$ increases beyond $v_{0,1}=$ $3 / 2$ in contradiction with (46) and (47). This result reflects the fact mentioned above that the RPM does not yield the energy $E_{1}$ for $v_{0}$ close to threshold.

If we repeat the calculation for the even states we obtain a perturbation ex- 
pansion with the expected slope at threshold:

$$
E=-\frac{1}{8} \xi+\frac{17}{192} \xi^{2}-\frac{23}{11520} \xi^{3}-\frac{271933}{19353600} \xi^{4}+\frac{29363423}{8128512000} \xi^{5}+O\left(\xi^{6}\right)
$$

At first sight, this result is surprising because no new even state should appear when $3 / 2<v_{0}<4$ (the ground state remains bound for all $v_{0}>0$ ). The explanation is that the RPM yields a spurious even-state energy associated to the divergent solution $\psi_{1,0}$. For example, when $v_{0}=1.51$ the RPM with $D=10$ and $d=0$ yields the actual ground-state energy $E_{0} \approx-0.70483$ and the spurious root $W \approx-0.00124114797000675832$. The considerably greater accuracy of the latter is due to the fact that the RPM yields the exact result when $v_{0}=3 / 2$. The question remains why the RPM does not yield the energy $E_{1}$ of the odd state in view of the fact that the calculation of the critical parameter is also exact in this case. The perturbation expansion (48) with the wrong slope at threshold also describes a spurious root. For example, when $v_{0}=1.49$ the roots of the Hankel determinants with $D=10$ and $d=0$ yields $W \approx-0.0012609753609799139195(s=1)$ and $E_{0} \approx-0.692231(s=$ 0 ). It is clear that the RPM favours the unphysical solutions; in fact, it is also interesting that the spurious roots of the Hankel determinants follow the unphysical expansions (49) and (48) for both $\xi<0$ and $\xi>0$ and in either case the rate of convergence of the corresponding sequences is remarkably large. For example, the expansion (49) predicts a positive root for a negative value of $\xi$ and the RPM already yields it quite accurately. When $v_{0}=1.49$ we obtain $W=0.0012588560223263235359$ in agreement with that perturbation series.

The second excited state vanishes when $v_{0}=4$. The RPM perturbation expansions obtained by substitution of $v_{0}=4+\xi$ are also unphysical. For example when $s=0$ we obtain a series with the wrong slope at threshold:

$$
E=\frac{1}{32} \xi-\frac{23}{2304} \xi^{2}-\frac{919}{331776} \xi^{3}+\frac{100843}{59719680} \xi^{4}-\frac{418250431}{1203948748800} \xi^{5}+O\left(\xi^{6}\right) .
$$


This results is not surprising if we take into account that the RPM fails to give us the second excited state when $v_{0}$ is slightly larger than 4 . When $s=1$ we obtain the exact power series for another unphysical root of the Hankel determinants

$$
E=-\frac{1}{32} \xi+\frac{7}{768} \xi^{2}+\frac{1921}{552960} \xi^{3}-\frac{1186027}{696729600} \xi^{4}+\frac{2551967839}{14046068736000} \xi^{5}+O\left(\xi^{6}\right)
$$

From the roots of the Hankel determinants for $v_{0}=4.01$ we obtain $E_{1} \approx$ -0.429395 (the actual energy for the first-excited state) and the spurious eigenvalue $W \approx-0.00031158508464057747545$ which is associated to the divergent function $\psi_{2,1}$ when $\xi \rightarrow 0\left(E \rightarrow 0^{-}\right)$.

For some unknown reason the RPM yields the unphysical roots associated to the divergent states more accurately than the physical ones stemming from the convergent states. However, the approach is still a useful tool for obtaining the eigenvalues and critical parameters of one-dimensional wells as already shown above. The modified RPM discussed in section 5 also yields the same spurious roots; therefore, we may conclude that such an unexpected behaviour is inherent in the Hankel determinants constructed from either the coefficients $f_{j}$ or $g_{j}$.

In an attempt to understand the baffling results discussed above we analyzed the zeroes of the denominators of the Padé approximants for energies in the neighborhoods of the actual eigenvalues and the spurious ones. However, we could not derive any reasonable rule from such study.

Although the RPM yields the whole set of critical values with either the even or odd functions for the modified Pöschl-Teller potential, the Hankel determinants do not exhibit spurious roots in this case. 


\section{Conclusions}

Despite of being a local approximation the RPM may be a useful tool for the calculation of critical parameters of one-dimensional and central-field quantum wells. In some cases, like the modified Pöschl-Teller and rational potentials, the original version of the approach yields accurate results. In other cases, like the Gaussian potential, it is necessary to resort to a modified algorithm that applies to one-dimensional models as well as to the s-states of centralfield ones. The accuracy of the estimated critical parameters is satisfactory for the Gaussian and Yukawa potentials. The original RPM proves to be suitable for states with $l>0$ and its accuracy increases with this quantum number. For this reason the RPM appears to be a powerful tool for the calculation of critical parameters for extremely large values of the angular-momentum quantum number.

In the case of the modified Pöschl-Teller and rational potentials the RPM yields the whole set of critical parameters for both the even and odd solutions to the Schrödinger equation. Half of them are associated to the divergent solutions. The occurrence of unphysical results is due to the fact that the RPM does not take into account the asymptotic behaviour of the eigenfunctions explicitly. For some unknown reason the RPM seems to favour the divergent solutions in the case of the rational potential. This undesirable behaviour is not a serious limitation because there is no doubt about which roots are spurious. We have not been able to give a sound answer to this anomalous behavior from the roots of the denominator of the Padé approximants.

The Pöschl-Teller and rational potentials are different in the sense that the RPM yields accurate energies close to the threshold in the former case but not in the latter one. Therefore, the three one-dimensional potentials discussed in section 4 reveal three different behaviour patterns in the application of the RPM to simple one-dimensional parity-invariant quantum wells. 
The RPM yields the exact solutions for the rational potential (23) when $E=0$. This particularly fortunate situation enables one to try perturbation theory about the threshold. Surprisingly, the RPM yields exact perturbation series only for the unphysical case of divergent functions. The reason may be that the physically meaningful solutions do not exhibit power-series expansions about threshold. However, we know that such expansions exist in the case of the Pöschl-Teller potential as shown in Eq. (44). In this case the Hankel determinants do not exhibit spurious roots.

We have also carried out calculations for potentials of the form $V(x)=$ $-v_{0} /\left(1+x^{2}\right)^{m}$, where $m=5 / 2,3,4$. In these cases the RPM fails to provide the critical parameters and the modified RPM exhibits convergent roots. The even and odd critical parameters appear separately as in the case of the Gaussian potential discussed in subsection 4.2 .

\section{Appendix}

If we change the independent and dependent variables in the Schrödinger equation with the rational potential (23) according to

$$
\begin{aligned}
x & =i z, \\
\varphi(z) & =\psi(i z)=\sqrt{1-z^{2}} w(z),
\end{aligned}
$$

then we obtain

$$
\frac{d}{d z}\left(1-z^{2}\right) \frac{d}{d z} w+\left[-2 E\left(1-z^{2}\right)-\frac{2 v_{0}+1}{1-z^{2}}\right] w=0
$$

This equation is a particular case of the spheroidal differential equation

$$
\frac{d}{d z}\left(1-z^{2}\right) \frac{d}{d z} w+\left[\lambda+\gamma^{2}\left(1-z^{2}\right)-\frac{\mu^{2}}{1-z^{2}}\right] w=0
$$


with $\lambda=0, \gamma^{2}=-2 E$ and $\mu^{2}=2 v_{0}+1$. We can also relate equation (53) with the associated Legendre equation

$$
\frac{d}{d z}\left(1-z^{2}\right) \frac{d}{d z} w+\left[\nu(\nu+1)-\frac{\mu^{2}}{1-z^{2}}\right] w=0
$$

when $E=0$ and $\nu=0$.

However, for the present discussion we prefer to proceed in a different way. The rational potential (23) exhibits singularities at $x= \pm i$. If we substitute

$$
\psi(x)=\left(1+x^{2}\right)^{\alpha} u(x)
$$

into the Schrödinger equation we obtain an eigenvalue equation for the new dependent variable $u(x)$ :

$$
\begin{aligned}
\left(1+x^{2}\right) u^{\prime \prime} & +4 \alpha x u^{\prime}-\frac{2\left(2 \alpha^{2}-2 \alpha-v_{0}\right)}{1+x^{2}} u \\
& +\left[2 E x^{2}+2\left(2 \alpha^{2}-\alpha+E\right)\right] u=0 .
\end{aligned}
$$

We remove the third term by choosing $\alpha$ to be any one of the roots of

$$
2 \alpha^{2}-2 \alpha-v_{0}=0
$$

Then, we expand $u$ in a Taylor series about the origin

$$
u(x)=\sum_{j=0}^{\infty} c_{j} x^{2 j+s}
$$

and derive a three-term recurrence relation for the coefficients

$$
\begin{aligned}
(2 j+s+1)(2 j+s+2) c_{j+1} & +[(2 j+s+2 \alpha)(2 j+s+2 \alpha-1)+2 E] c_{j} \\
+ & 2 E c_{j-1}=0 .
\end{aligned}
$$

The radius of convergence of this series is unity $\left(\lim _{j \rightarrow \infty}\left|\frac{c_{j+1}}{c_{j}}\right|=1\right)$ because of the singularities at $x= \pm i$. When $E=0$ the recurrence relation becomes a 
two-term one and we can obtain polynomial solutions for particular values of $\alpha$. Note that if

$$
\alpha=\left\{\begin{array}{c}
\alpha_{1}(k, s)=-k-\frac{s}{2} \\
\alpha_{2}(k, s)=-k-\frac{s}{2}+1
\end{array}\right.
$$

then $c_{j}=0$ for all $j>k$.We thus have two sets of critical potential parameters:

$$
v_{0}=\frac{(2 \alpha-1)^{2}-1}{2}=\left\{\begin{array}{l}
v_{0}^{(1)}(k, s)=\frac{(2 k+s-1)^{2}-1}{2} \\
v_{0}^{(2)}(k, s)=\frac{(2 k+s)^{2}-1}{2}
\end{array}\right.
$$

where $v_{0}^{(1,2)}=v_{0}\left(\alpha_{1,2}\right)$. It is interesting to note that the critical parameters exhibit a kind of degeneracy:

$$
\begin{gathered}
v_{0}^{(1)}(k, 1)=v_{0}^{(2)}(k, 0)=\frac{4 k^{2}-1}{2}, k=1,2, \ldots, \\
v_{0}^{(1)}(k+1,0)=v_{0}^{(2)}(k, 1)=\frac{(2 k+1)^{2}-1}{2}, k=1,2, \ldots,
\end{gathered}
$$

similar to the one found by Joseph [20,24] for the central-field version of this model. In the present case one of the degenerate solutions is convergent $(\sim 1)$ and the other one is divergent $(\sim x)$. The connection between both models becomes apparent if we take into account that the states of the central-field model with angular-momentum quantum numbers $l=-1$ and $l=0$ become the even and odd states of the one-dimensional one.

In order to understand the results derived in the subsection 4.3 by means of the RPM simply note that

$$
f(x)=\frac{s}{x}-\frac{\psi^{\prime}(x)}{\psi(x)}=\frac{s}{x}-\frac{2 \alpha x}{1+x^{2}}-\frac{u^{\prime}(x)}{u(x)},
$$

is a rational function of $x$. 


\section{References}

[1] V. Bargmann, On the number of bound states in a central field of force, Proc. Nat. Acad. Sci. USA 38:961-966 (1952).

[2] J. Schwinger, On the bound states of a given potential, Proc. Nat. Acad. Sci. USA 47:122-129 (1961).

[3] G. C. Ghirardi and A. Rimini, On the number of bound states of a given interaction, J. Math. Phys. 6:40-44 (1965).

[4] F. Calogero, Sufficient conditions for an attractive potential to possess bound states, J. Math. Phys. 6:161-164 (1965).

[5] F. Calogero, Sufficient conditions for an attractive potential to possess bound states. II, J. Math. Phys. 6:1105-1107 (1965).

[6] R. G. Newton, Bounds on the number of bound states for the Schrödinger equation in one and two dimensions, J. Operator Theory 10:119-125 (1983).

[7] K. Chadan, A. Martin, and J. Stubbe, The Calogero bound for nonzero angular momentum, J. Math. Phys. 36:1616-1624 (1995).

[8] K. Chadan, R. Kobayashi, A. Martin, and J. Stubbe, Generalization of the Calogero-Cohn bound on the number of bound sates, J. Math. Phys. 37:11061114 (1996).

[9] K. Chadan and R. Kobayashi, A sufficient condition for the existence of bound states in a potential, J. Math. Phys. 38:4900-4908 (1997).

[10] F. Brau and F. Calogero, Upper and lower limits for the number of s-wave bound states in an attractive potential, J. Math. Phys. 44:1554-1575 (2003).

[11] F. Brau and F. Calogero, Upper and lower limits on the number of bound states in a central potential, J. Phys. A 36:12021-12063 (2003).

[12] F. Brau and F. Calogero, Lower limit in semiclassical form for the number of bound states in a central potential, Phys. Lett. A 321:225-230 (2004). 
[13] Z-Q. Ma, The Levinson theorem, J. Phys. A 39:R625-R659 (2006).

[14] E. Z. Liverts and N. Barnea, Transition states and the critical parameters of central potentials, J. Phys. A 44:375303 (2011).

[15] F. M. Fernández, Q. Ma, and R. H. Tipping, Tight upper and lower bounds for energy eigenvalues of the Schrödinger equation, Phys. Rev. A 39:1605-1609 (1989).

[16] F. M. Fernández, Q. Ma, and R. H. Tipping, Eigenvalues of the Schrödinger equation via the Riccati-Padé method, Phys. Rev. A 40:6149-6153 (1989).

[17] S. Abbasbandy and C. Bervillier, Analytic continuation of Taylor series and the boundary value problems of some nonlinear ordinary differential equations, Appl. Math. Comput. 218:2178-2199 (2011).

[18] P. Amore and F. M. Fernández, Rational Approximation to the Solutions of Two-Point Boundary Value Problems, Acta Polytech. 51:9-13 (2011). arXiv:0705.3862 [math-ph].

[19] S. Flügge, Practical Quantum Mechanics. Springer-Verlag, Berlin, 1999.

[20] A. Joseph, On the determination of the exact number of bound states of a given potential I. General method, Int. J. Quantum Chem. 1:615-629 (1967).

[21] D. Singh and Y. P. Varshni, Comparative study of the bound states of static screened Coulomb and cut-off potentials, Phys. Rev. A 29:2895-2898 (1984).

[22] R. P. Feynman, Forces in Molecules, Phys. Rev. 56:340-343 (1939).

[23] F. M. Fernández, Comment on "Breakdown of the Hellmann-Feynman theorem: Degeneracy is the key", Phys. Rev. B 69:037101 (2004)

[24] A. Joseph, The theory of local degeneracy, Int. J. Quantum Chem. 1:535-559 (1967).

[25] F. M. Fernández and J. Garcia, Comment on 'A simple analytical expression for bound state energies for an attractive Gaussian confining potential', Phys. Scr. 87:027001 (2013). 
Table 1

Critical parameters for the Gaussian Well

\begin{tabular}{|c|c|c|}
\hline $\mathrm{n}$ & $v_{0, n}$ (present) & $\beta / 2 \quad 14$ \\
\hline 1 & 1.34200232546204576914 & 1.3420023 \\
\hline 2 & 4.32454875170105636793 & \\
\hline 3 & 8.89784977356695359410 & 8.89785 \\
\hline 4 & 15.05314025436583553157 & \\
\hline 5 & 22.78673996005213242180 & 22.78674 \\
\hline 6 & 32.09666656038554309293 & \\
\hline 7 & 42.98170019005867752947 & 42.9817 \\
\hline 8 & 55.44102390556364979485 & \\
\hline 9 & 69.47405735384177416019 & 69.47406 \\
\hline 10 & 85.08036985819273906133 & \\
\hline 11 & 102.2596308675957148370 & 102.25963 \\
\hline 12 & 121.0115797852355989558 & \\
\hline 13 & 141.3360066230547124163 & 141.33601 \\
\hline 14 & 163.2327390694287387382 & \\
\hline 15 & 186.7016335410971417677 & 186.70163 \\
\hline 16 & 211.7425688100812690936 & \\
\hline 17 & 238.3554413511863843581 & 238.35544 \\
\hline 18 & 266.5401618724453436776 & \\
\hline 19 & 296.2966526792028587502 & 296.29665 \\
\hline 20 & 327.6248456385162999270 & \\
\hline 21 & 360.5246805841777739108 & 360.52468 \\
\hline
\end{tabular}


Table 2

Critical parameters for the Yukawa potential: s states

\begin{tabular}{|l|c|c|c|}
\hline State & $v_{0, n}$ (present) & $\beta / 2[14]$ & $1 / \delta[21]$ \\
\hline $1 s$ & 0.83990388669822801527775556 & 0.83990390 & 0.839903886698226 \\
$2 s$ & 3.2236301610682666483973 & 3.2236302 & 3.22363017 \\
$3 s$ & 7.17101392084392858317 & 7.1710140 & 7.17101392 \\
$4 s$ & 12.68582992202390726756 & 12.685830 & 12.685830 \\
$5 s$ & 19.76942118485633321537 & 19.769421 & 19.769421 \\
$6 s$ & 28.42243219866087345719 & 28.42243 & 28.422432 \\
$7 s$ & 38.64522743052775121132 & 38.645227 & 38.645227 \\
\hline
\end{tabular}


Table 3

Critical parameters for the Yukawa potential: states with $l>0$

\begin{tabular}{|c|c|c|c|c|}
\hline$l$ & $n$ & $\mathrm{RPM}$ & $\beta_{n, l} / 2[14]$ & $1 / \delta[21]$ \\
\hline 1 & 1 & 4.540979480 & 4.5409795 & 4.540979547 \\
\hline 1 & 2 & 8.872287943 & 8.872288 & 8.87228793 \\
\hline 1 & 3 & 14.7307131 & 14.730713 & 14.730713 \\
\hline 1 & 4 & 22.1306205 & 22.130627 & 22.130627 \\
\hline 2 & 1 & 10.947492231128 & 10.947492 & 10.947492 \\
\hline 2 & 2 & 17.21020724698 & 17.210207 & 17.210207 \\
\hline 2 & 3 & 24.98478805031 & 24.984788 & 24.984788 \\
\hline 2 & 4 & 34.285733608 & 34.2857335 & 34.285734 \\
\hline 3 & 1 & 20.06777597598021672 & 20.067776 & 20.067776 \\
\hline 3 & 2 & 28.257056865147125 & 28.257057 & 28.257057 \\
\hline 3 & 3 & 37.949696830060 & 37.949697 & 37.949696 \\
\hline 3 & 4 & 49.1589622686 & 49.1589625 & 49.158964 \\
\hline 4 & 1 & 31.904488236447390251 & 31.904488 & 31.904488 \\
\hline 4 & 2 & 42.01838864622171175 & 42.0183885 & 42.018390 \\
\hline 4 & 3 & 53.6301861108720125 & 53.630185 & 53.630187 \\
\hline 4 & 4 & 66.7518302698487 & 66.75183 & 66.751829 \\
\hline 5 & 1 & 46.458582142052657720625 & 46.458582 & 46.458583 \\
\hline 5 & 2 & 58.4961723904053473472 & 58.49617 & 58.496171 \\
\hline 5 & 3 & 72.02784452443215966 & 72.027845 & 72.027848 \\
\hline 5 & 4 & 87.0643772674642755 & 87.064375 & \\
\hline
\end{tabular}


Table 4

Critical parameters for the gaussian well: states with $l>0$

\begin{tabular}{|c|c|c|c|}
\hline$l$ & $n$ & $\mathrm{RPM}$ & $\beta_{n, l} / 2$ [14] \\
\hline 1 & 1 & 6.049654263 & 6.0496545 \\
\hline 1 & 2 & 17.544888 & 17.544888 \\
\hline 1 & 3 & 35.241431 & 35.241428 \\
\hline 1 & 4 & 59.17581 & 59.175825 \\
\hline 2 & 1 & 13.4505387996 & 13.450539 \\
\hline 2 & 2 & 28.83788607 & 28.837886 \\
\hline 2 & 3 & 50.35752508 & 50.357525 \\
\hline 2 & 4 & 78.063746 & 78.063745 \\
\hline 3 & 1 & 23.553930851605 & 23.553931 \\
\hline 3 & 2 & 42.81369669354 & 42.813696 \\
\hline 3 & 3 & 68.162501708 & 68.162500 \\
\hline 3 & 4 & 99.65923348 & 99.659230 \\
\hline 4 & 1 & 36.366501836074 & 36.366502 \\
\hline 4 & 2 & 59.48855740034 & 59.488555 \\
\hline 4 & 3 & 88.6698082860 & 88.669810 \\
\hline 4 & 4 & 123.9690574563 & 123.96905 \\
\hline
\end{tabular}


Table 5

Critical parameters for the Yukawa potential with $n=1$ and large $l$ calculated by means of the RPM, WKB and variational approaches. LE is the logarithmic error: $\log \mid($ exact - approximate) $/$ exact $\mid$

\begin{tabular}{|c|c|c|c|c|c|}
\hline$l$ & RPM & WKB [14] & LE & Variational & LE \\
\hline 50 & 3514.7478136194717430 & 3466 & -1.9 & 3518 & -3.1 \\
100 & 13824.314996806666238 & 13727 & -2.2 & 13830 & -3.4 \\
150 & 30929.586489910790437 & 30785 & -2.3 & 30938 & -3.5 \\
200 & 54830.562488458876745 & 54637 & -2.5 & 54842 & -3.7 \\
250 & 85527.243031809696017 & 85286 & -2.5 & 85542 & -3.8 \\
300 & 123019.62813311806352 & 122730 & -2.6 & 123037 & -3.8 \\
350 & 167307.71779803038796 & 166970 & -2.7 & 167328 & -3.9 \\
400 & 218391.51202937270652 & 218006 & -2.8 & 218415 & -4.0 \\
450 & 276271.01082871615922 & 275838 & -2.8 & 276297 & -4.0 \\
500 & 340946.21419700393386 & 340465 & -2.9 & 340975 & -4.1 \\
\hline
\end{tabular}


Table 6

Critical parameters for the Gaussian potential with $n=1$ and large $l$ calculated by means of the RPM, WKB and variational approaches. LE is the logarithmic error: $\log \mid($ exact - approximate) / exact $\mid$

\begin{tabular}{|c|c|c|c|c|c|}
\hline$l$ & RPM & WKB [14] & LE & Variational & LE \\
\hline 50 & 3563.4739040116520856 & 3466 & -1.6 & 3570 & -2.8 \\
100 & 13921.096733881441676 & 13727 & -1.9 & 13933 & -3.1 \\
150 & 31074.422038210807820 & 30785 & -2.0 & 31092 & -3.2 \\
200 & 55023.451386983337338 & 54637 & -2.2 & 55047 & -3.4 \\
250 & 85768.185095826709503 & 85286 & -2.3 & 85798 & -3.5 \\
300 & 123308.62327017069510 & 122730 & -2.3 & 123344 & -3.5 \\
350 & 167644.76595525290586 & 166970 & -2.4 & 167686 & -3.6 \\
400 & 218776.61317370956855 & 218006 & -2.5 & 218824 & -3.7 \\
450 & 276704.16493812321886 & 275838 & -2.5 & 276757 & -3.7 \\
500 & 341427.42125604645356 & 340465 & -2.5 & 341486 & -3.8 \\
\hline
\end{tabular}

